\title{
First-order impulsive differential systems: sufficient and necessary conditions for oscillatory or asymptotic behavior
}

\author{
Shyam Sundar Santra ${ }^{1 *}$ (D) Dumitru Baleanu ${ }^{2,3,4}$, Khaled Mohamed Khedher ${ }^{5,6}$ and Osama Moaaz
}

\section{Correspondence:}

shyam01.math@gmail.com

'Department of Mathematics, JIS

College of Engineering, Kalyani

741235, India

Full list of author information is

available at the end of the article

\section{Springer}

\begin{abstract}
In this paper, we study the oscillatory and asymptotic behavior of a class of first-order neutral delay impulsive differential systems and establish some new sufficient conditions for oscillation and sufficient and necessary conditions for the asymptotic behavior of the same impulsive differential system. To prove the necessary part of the theorem for asymptotic behavior, we use the Banach fixed point theorem and the Knaster-Tarski fixed point theorem. In the conclusion section, we mention the future scope of this study. Finally, two examples are provided to show the defectiveness and feasibility of the main results.
\end{abstract}

MSC: $34 \mathrm{C} 10 ; 34 \mathrm{~K} 11$

Keywords: Impulsive; Knaster-Tarski fixed point theorem; Oscillation; Delay; Neutral; Banach fixed point theorem

\section{Introduction}

Nowadays impulsive differential systems are attracting a lot of attention. They appear in several real world problems (see, for instance, [1-3]). In general, it is well known that several natural phenomena are driven by differential equations. However, the description of some real world problems requires studies on impulsive differential systems, a subject very interesting from the mathematical point of view. Examples of the aforementioned phenomena are related to theoretical physics, pharmacokinetics, population dynamics, biotechnology processes, biological systems, mechanical systems, control theory, chemistry, engineering (we also stress that the modeling of these phenomena is suitably formulated by evolutive partial differential equations and, moreover, moment problem approaches appear also as a natural instrument in control theory of neutral type systems; see [4-6] and [7-9], respectively).

The literature related to impulsive differential equations is very wide. Here we mention some recent developments in this field.

(c) The Author(s) 2021. This article is licensed under a Creative Commons Attribution 4.0 International License, which permits use sharing, adaptation, distribution and reproduction in any medium or format, as long as you give appropriate credit to the original author(s) and the source, provide a link to the Creative Commons licence, and indicate if changes were made. The images or other third party material in this article are included in the article's Creative Commons licence, unless indicated otherwise in a credit line to the material. If material is not included in the article's Creative Commons licence and your intended use is not permitted by statutory regulation or exceeds the permitted use, you will need to obtain permission directly from the copyright holder. To view a copy of this licence, visit http://creativecommons.org/licenses/by/4.0/. 
In [10], Shen and Wang considered impulsive differential systems of the following form:

$$
\left\{\begin{array}{l}
v^{\prime}(\xi)+b_{1}(\xi) v\left(\xi-\mu_{1}\right)=0, \quad \xi \neq \alpha_{i}, \xi \geq \xi_{0} \\
v\left(\alpha_{i}^{+}\right)-v\left(\alpha_{i}^{-}\right)=I_{i}\left(v\left(\alpha_{i}\right)\right), \quad i \in \mathbb{N}
\end{array}\right.
$$

where $b \in C(\mathbb{R}, \mathbb{R})$ and $I_{i} \in C(\mathbb{R}, \mathbb{R})$ for $i \in \mathbb{N}$, and established some sufficient conditions that ensure the oscillatory and asymptotic behavior of (1).

In [11], Graef et al. studied the impulsive system

$$
\left\{\begin{array}{l}
(v(\xi)-b(\xi) v(\xi-\mu))^{\prime}+b_{1}(\xi)\left|v\left(\xi-\mu_{1}\right)\right|^{\lambda} \operatorname{sgn} v\left(\xi-\mu_{1}\right)=0, \quad \xi \geq \xi_{0} \\
v\left(\alpha_{i}^{+}\right)=b_{i} v\left(\alpha_{i}\right), \quad i \in \mathbb{N}
\end{array}\right.
$$

assuming that $b(\xi) \in \operatorname{PC}\left(\left[\xi_{0}, \infty\right), \mathbb{R}_{+}\right)$(that is, $b(\xi)$ is piecewise continuous in $\left.\left[\xi_{0}, \infty\right)\right)$, established some new sufficient conditions for the oscillation of (2).

In [12], the authors established some new oscillation criteria for first order impulsive neutral delay differential systems of the form

$$
\left\{\begin{array}{l}
(v(\xi)-b(\xi) v(\xi-\mu))^{\prime}+b_{1}(\xi) v\left(\xi-\mu_{1}\right)-b_{2}(\xi) v\left(\xi-\mu_{2}\right)=0, \quad \mu_{1} \geq \mu_{2}>0 \\
v\left(\alpha_{i}^{+}\right)=I_{i}\left(v\left(\alpha_{i}\right)\right), \quad i \in \mathbb{N},
\end{array}\right.
$$

under the assumptions that $b(\xi) \in \operatorname{PC}\left(\left[\xi_{0}, \infty\right), \mathbb{R}_{+}\right)$and $b_{i} \leq \frac{I_{i}(v)}{v} \leq 1$.

Karpuz et al. in [13] extended the results contained in [12] by taking the nonhomogeneous counterpart of system (3) with variable delays.

Oscillation and non-oscillation properties of second-order linear neutral differential equations with impulses were studied by Tripathy and Santra in [14], where the authors considered the problem

$$
\left\{\begin{array}{l}
(v(\xi)-b v(\xi-\mu))^{\prime \prime}+b_{1} v\left(\xi-\mu_{1}\right)=0, \quad \xi \neq \alpha_{i}, i \in \mathbb{N} \\
\Delta\left(v\left(\alpha_{i}\right)-\tilde{b} v\left(\alpha_{i}-\mu\right)\right)^{\prime}+b_{2} v\left(\alpha_{i}-\mu_{1}\right)=0, \quad i \in \mathbb{N}
\end{array}\right.
$$

where all coefficients and delays are constant. Other sufficient and necessary conditions for the oscillatory or asymptotic behavior of second-order neutral delay differential equations with impulses were obtained in [15], where Tripathy and Santra studied systems of the form

$$
\left\{\begin{array}{l}
\left(p(\xi)(v(\xi)+b(\xi) v(\xi-\mu))^{\prime}\right)^{\prime}+b_{1}(\xi) g\left(v\left(\xi-\mu_{1}\right)\right)=0, \quad \xi \neq \alpha_{i}, i \in \mathbb{N} \\
\Delta\left(p\left(\alpha_{i}\right)\left(v\left(\alpha_{i}\right)+b\left(\alpha_{i}\right) v\left(\alpha_{i}-\mu\right)\right)^{\prime}\right)+b_{2}\left(\alpha_{i}\right) g\left(v\left(\alpha_{i}-\mu_{1}\right)\right)=0, \quad i \in \mathbb{N}
\end{array}\right.
$$

In [15], in particular, the authors were interested in oscillating systems that, after a perturbation by instantaneous change of state, remain oscillating. 
In [16], Santra and Tripathy investigated the oscillatory or asymptotic behavior of the solutions for first-order neutral delay differential system

$$
\left\{\begin{array}{l}
(v(\xi)-b(\xi) v(\xi-\mu))^{\prime}+b_{1}(\xi) g\left(v\left(\xi-\mu_{1}\right)\right)=0, \quad \xi \neq \alpha_{i}, \xi \geq \xi_{0} \\
v\left(\alpha_{i}^{+}\right)=I_{i}\left(v\left(\alpha_{i}\right)\right), \quad i \in \mathbb{N}, \\
v\left(\alpha_{i}^{+}-\mu_{1}\right)=I_{i}\left(v\left(\alpha_{i}-\mu_{1}\right)\right), \quad i \in \mathbb{N}
\end{array}\right.
$$

for different values of the neutral coefficient $b$.

We also mention the paper [17] in which Santra and Dix, using the Lebesgue dominated convergence theorem, obtained sufficient and necessary conditions for the oscillation of the following second-order neutral differential equations with impulses:

$$
\left\{\begin{array}{l}
\left(p(\xi)\left(h^{\prime}(\xi)\right)^{\gamma}\right)^{\prime}+\sum_{j=1}^{m} r_{j}(\xi) g_{j}\left(v\left(\tilde{\mu}_{j}(\xi)\right)\right)=0, \quad \xi \geq \xi_{0}, \xi \neq \alpha_{i}, i \in \mathbb{N}, \\
\Delta\left(p\left(\alpha_{i}\right)\left(h^{\prime}\left(\alpha_{i}\right)\right)^{\gamma}\right)+\sum_{j=1}^{m} \widetilde{r}_{j}\left(\alpha_{i}\right) g_{j}\left(v\left(\tilde{\mu}_{j}\left(\alpha_{i}\right)\right)\right)=0,
\end{array}\right.
$$

where

$$
h(\xi)=v(\xi)+b(\xi) v(\mu(\xi)), \quad \Delta v(\xi)=\lim _{\eta \rightarrow \xi^{+}} v(\eta)-\lim _{\eta \rightarrow \xi^{-}} v(\eta), \quad-1 \leq b(\xi) \leq 0 .
$$

In line with the contents of [17], Tripathy and Santra in [18] examined oscillation and non-oscillation properties for the solutions of the following forced nonlinear neutral impulsive differential system:

$$
\left\{\begin{array}{l}
\left(p(\xi)(u(\xi)+b(\xi) v(\xi-\mu))^{\prime}\right)^{\prime}+r(\xi) g\left(u\left(\xi-\mu_{1}\right)\right)=f(\xi), \quad \xi \neq \alpha_{i}, i \in \mathbb{N} \\
\Delta\left(p\left(\alpha_{i}\right)\left(v\left(\alpha_{i}\right)+b\left(\alpha_{i}\right) v\left(\alpha_{i}-\mu\right)\right)^{\prime}\right)+\tilde{r}\left(\alpha_{i}\right) g\left(v\left(\alpha_{i}-\mu_{1}\right)\right)=\tilde{f}\left(\alpha_{i}\right), \quad i \in \mathbb{N}
\end{array}\right.
$$

for different values of $b(\xi)$ and established sufficient conditions for the existence of positive bounded solutions of system (8).

Finally we mention the recent work [19] in which Tripathy and Santra studied the characterizations for the oscillation of second-order neutral delay impulsive differential system

$$
\left\{\begin{array}{l}
\left(p(\xi)\left(h^{\prime}(\xi)\right)^{\gamma}\right)^{\prime}+\sum_{j=1}^{m} \tilde{b}_{j}(\xi) v^{\alpha_{j}}\left(\tilde{\mu}_{j}(\xi)\right)=0, \quad \xi \geq \xi_{0}, \xi \neq \alpha_{i}, \\
\Delta\left(p\left(\alpha_{i}\right)\left(h^{\prime}\left(\alpha_{i}\right)\right)^{\gamma}\right)+\sum_{j=1}^{m} \tilde{b}_{j}\left(\alpha_{i}\right) v^{\alpha_{j}}\left(\tilde{\mu}_{j}\left(\alpha_{i}\right)\right)=0, \quad i \in \mathbb{N},
\end{array}\right.
$$

where $h(\xi)=v(\xi)+b(\xi) v(\mu(\xi))$ and $-1<b(\xi) \leq 0$.

For further details on neutral impulsive differential equations and for recent results related to the oscillation theory for delay differential equations, we refer the reader to the papers [20-53] and to the references therein. In particular, the study of oscillation of halflinear/Emden-Fowler (neutral) differential equations with deviating arguments (delayed or advanced arguments or mixed arguments) has numerous applications in physics and engineering (e.g., half-linear/Emden-Fowler differential equations arise in a variety of real world problems such as in the study of $p$-Laplace equations, chemotaxis models, and so forth); see, e.g., the papers $[4,44-47,49,50,52,53]$ for more details. In particular, by using different methods, the following papers were concerned with the oscillation of various classes of half-linear/Emden-Fowler differential equations and half-linear/EmdenFowler differential equations with different neutral coefficients (e.g., the paper [43] was 
concerned with neutral differential equations assuming that $0 \leq b(\xi)<1$ and $b(\xi)>1$; in [44] the authors studied neutral differential equations assuming that $0 \leq b(\xi)<1$; in [46], the authors considered neutral differential equations assuming that $b(\xi)$ is nonpositive; in $[47,51]$ the author considered neutral differential equations in the case where $b(\xi)>1$; the paper [50] was concerned with neutral differential equations assuming that $0 \leq b(\xi) \leq q_{0}<\infty$ and $b(\xi)>1$; in [52] the authors considered neutral differential equations in the case where $0 \leq b(\xi) \leq q_{0}<\infty$; in [53] the author studied neutral differential equations in the case when $0 \leq b(\xi)=b_{0} \neq 1$; whereas the paper [49] was concerned with differential equations with a nonlinear neutral term assuming that $0 \leq b(\xi) \leq a<1$ ), which is the same research topic as that of this paper.

Motivated by the aforementioned findings, in this paper we prove sufficient and necessary conditions for oscillatory or asymptotic behavior of solutions to a first-order nonlinear impulsive differential system in the form

$$
\left\{\begin{array}{l}
(v(\xi)+b(\xi) v(\xi-\mu))^{\prime}+b_{1}(\xi) G\left(v\left(\xi-\mu_{1}\right)\right)=f(\xi), \quad \xi \neq \alpha_{i}, i \in \mathbb{N} \\
\Delta\left(v\left(\alpha_{i}\right)+b\left(\alpha_{i}\right) v\left(\alpha_{i}-\mu\right)\right)+b_{2}\left(\alpha_{i}\right) G\left(v\left(\alpha_{i}-\mu_{1}\right)\right)=g\left(\alpha_{i}\right), \quad i \in \mathbb{N}
\end{array}\right.
$$

where

(a) $\mu>0, \mu_{1} \geq 0$ are real constants; $b_{1}, b_{2} \in C\left(\mathbb{R}_{+}, \mathbb{R}_{+}\right), b \in \mathrm{PC}\left(\mathbb{R}_{+}, \mathbb{R}\right)$;

(b) $f, g \in C(\mathbb{R}, \mathbb{R}), G \in C(\mathbb{R}, \mathbb{R})$ is nondecreasing with $v G(v)>0$ for $v \neq 0$;

(c) $\alpha_{i}$ for $i \in \mathbb{N}$ with $\alpha_{1}<\alpha_{2}<\cdots<\alpha_{i}<\ldots$ and $\lim _{i \rightarrow \infty} \alpha_{i}=\infty$ are fixed moments of impulsive effect;

(d) $\Delta$ is the difference operator defined by

$$
\Delta v(a)=\lim _{\eta \rightarrow a^{+}} v(\eta)-\lim _{\eta \rightarrow a^{-}} v(\eta)
$$

(e) there exists $F \in C(\mathbb{R}, \mathbb{R})$ such that $f(\xi)=F^{\prime}(\xi)$ and $g\left(\alpha_{i}\right)=\Delta F\left(\alpha_{i}\right), i \in \mathbb{N}$.

Next, we are listing all the assumptions/conditions which we need to study the oscillation and non-oscillation properties of the solution of system (E).

(A1) $\lim _{\xi \rightarrow \infty} F(\xi)=M,|M|<\infty$.

(A2) $F(\xi)$ changes sign with $-\infty<\liminf _{\xi \rightarrow \infty} F(\xi)<0<\limsup _{\xi \rightarrow \infty} F(\xi)<\infty$.

(A3) $F(\xi)$ changes sign with $F^{+}(\xi)=\max \{F(\xi), 0\}, F^{-}(\xi)=\max \{-F(\xi), 0\}$.

(A4) $G$ is odd with $G(u v)=G(u) G(v)$, and $G(u)+G(v) \geq \lambda G(u+v)$ for $u, v, \lambda>0$.

(A5) $\int_{\varsigma}^{\infty} B_{1}(\xi) G\left(F^{+}\left(\xi-\mu_{1}\right)\right) d \xi+\sum_{i=1}^{\infty} B_{2}\left(\alpha_{i}\right) G\left(F^{+}\left(\alpha_{i}-\mu_{1}\right)\right)=\infty$, where $\varsigma>0, \xi>\mu$, $B_{1}(\xi)=\min \left\{b_{1}(\xi), b_{1}(\xi-\mu)\right\}$ and $B_{2}\left(\alpha_{i}\right)=\min \left\{b_{2}\left(\alpha_{i}\right), b_{2}\left(\alpha_{i}-\mu\right)\right\}$.

(A6) $\int_{\varsigma}^{\infty} B_{1}(\xi) G\left(F^{-}\left(\xi-\mu_{1}\right)\right) d \xi+\sum_{i=1}^{\infty} B_{2}\left(\alpha_{i}\right) G\left(F^{-}\left(\alpha_{i}-\mu_{1}\right)\right)=\infty$, where $\varsigma>0$.

(A7) $\int_{\varsigma}^{\infty} b_{1}(\xi) G\left(F^{+}\left(\xi-\mu_{1}\right)\right) d \xi+\sum_{i=1}^{\infty} b_{2}\left(\alpha_{i}\right) G\left(F^{+}\left(\alpha_{i}-\mu_{1}\right)\right)=\infty$, where $\varsigma>0$.

(A8) $\int_{\varsigma}^{\infty} b_{1}(\xi) G\left(F^{-}\left(\xi-\mu_{1}\right)\right) d \xi+\sum_{i=1}^{\infty} b_{2}\left(\alpha_{i}\right) G\left(F^{-}\left(\alpha_{i}-\mu_{1}\right)\right)=\infty$, where $\varsigma>0$.

(A9) $\int_{\varsigma}^{\infty} b_{1}(\xi) G\left(F^{+}\left(\xi+\mu-\mu_{1}\right)\right) d \xi+\sum_{i=1}^{\infty} b_{2}\left(\alpha_{i}\right) G\left(F^{+}\left(\alpha_{i}+\mu-\mu_{1}\right)\right)=\infty$, where $\varsigma>0$.

(A10) $\int_{\varsigma}^{\infty} b_{1}(\xi) G\left(F^{-}\left(\xi+\mu-\mu_{1}\right)\right) d \xi+\sum_{i=1}^{\infty} b_{2}\left(\alpha_{i}\right) G\left(F^{-}\left(\alpha_{i}+\mu-\mu_{1}\right)\right)=\infty$, where $\varsigma>0$.

(A11) $G$ is superlinear and $\frac{G(u)}{u^{\gamma}} \geq \frac{G(v)}{v^{\gamma}}$ for $u \geq v$ and $\gamma>1$.

(A12) $\int_{\varsigma}^{\infty} \frac{b_{1}(\xi) G\left(F^{+}\left(\xi+\mu-\mu_{1}\right)\right)}{\left[F^{+}\left(\xi+\mu-\mu_{1}\right)\right]^{\gamma}} d \xi+\sum_{i=1}^{\infty} \frac{b_{2}\left(\alpha_{i}\right) G\left(F^{+}\left(\alpha_{i}+\mu-\mu_{1}\right)\right)}{c^{-} \gamma\left[F^{+}\left(\alpha_{i}+\mu-\mu_{1}\right)\right]^{\gamma}}=\infty$, where $\varsigma>0$ and $0<c \leq 1$.

(A13) $\int_{\varsigma}^{\infty} \frac{b_{1}(\xi) G\left(F^{-}\left(\xi+\mu-\mu_{1}\right)\right)}{\left[F^{-}\left(\xi+\mu-\mu_{1}\right)\right]^{\gamma}} d \xi+\sum_{i=1}^{\infty} \frac{b_{2}\left(\alpha_{i}\right) G\left(F^{-}\left(\alpha_{i}+\mu-\mu_{1}\right)\right)}{c^{-} \gamma\left[F^{-}\left(\alpha_{i}+\mu-\mu_{1}\right)\right]^{\gamma}}=\infty$, where $\varsigma>0$ and $0<c \leq 1$.

(A14) $\int_{0}^{\infty} b_{1}(\xi) d \xi+\sum_{i=1}^{\infty} b_{2}\left(\alpha_{i}\right)=\infty$. 


\section{Sufficient conditions for oscillation}

In this section, we establish sufficient conditions for the oscillation of the impulsive system (E).

Theorem 2.1 Under the assumptions $0 \leq b(\xi) \leq a<\infty$ for $\xi \in \mathbb{R}_{+}$and (A2)-(A6), every solution of system (E) is oscillatory.

Proof Let $v(\xi)$ be a solution of (E). For the sake of contradiction, let the solution be nonoscillatory. So, there exists $\xi_{0}>\rho=\max \left\{\mu, \mu_{1}\right\}$ such that $v(\xi)>0, v(\xi-\mu)>0$ and $v(\xi-$ $\left.\mu_{1}\right)>0$ for $\xi \geq \xi_{0}$. Setting

$$
\begin{aligned}
& h(\xi)=v(\xi)+b(\xi) v(\xi-\mu), \quad \xi \neq \alpha_{i}, i \in \mathbb{N} \\
& h\left(\alpha_{i}\right)=v\left(\alpha_{i}\right)+b\left(\alpha_{i}\right) v\left(\alpha_{i}-\mu\right), \quad i \in \mathbb{N},
\end{aligned}
$$

and

$$
H(\xi)=h(\xi)-F(\xi), \quad H\left(\alpha_{i}\right)=h\left(\alpha_{i}\right)-F\left(\alpha_{i}\right),
$$

it follows from (E) that

$$
\begin{aligned}
& H^{\prime}(\xi)=-b_{1}(\xi) G\left(v\left(\xi-\mu_{1}\right)\right) \leq 0, \quad \xi \neq \alpha_{i}, i \in \mathbb{N}, \\
& \Delta H\left(\alpha_{i}\right)=-b_{2}\left(\alpha_{i}\right) G\left(v\left(\alpha_{i}-\mu_{1}\right)\right) \leq 0, \quad i \in \mathbb{N},
\end{aligned}
$$

for $\xi \geq \xi_{1}>\xi_{0}+\mu_{1}$. Consequently, $H(\xi)$ is nonincreasing and monotonic on $\left[\xi_{2}, \infty\right)$, where $\xi_{2}>\xi_{1}$. So, we have the following two possible cases.

Case (i). Let $H(\xi)<0$ for $\xi \geq \xi_{2}$. Since $h(\xi)>0$, then $F(\xi)>0$ for $\xi \geq \xi_{2}$, which is a contradiction.

Case (ii). Let $H(\xi)>0$ for $\xi \geq \xi_{2}$. Ultimately, $h(\xi)>F(\xi)$ and hence $h(\xi)>\max \{0, F(\xi)\}=$ $F^{+}(\xi)$ for $\xi \geq \xi_{2}$. Using (11), the first equation of system (E) becomes

$$
0=H^{\prime}(\xi)+b_{1}(\xi) G\left(v\left(\xi-\mu_{1}\right)\right)+G(a)\left[H^{\prime}(\xi-\mu)+b_{1}(\xi-\mu) G\left(v\left(\xi-\mu-\mu_{1}\right)\right)\right]
$$

for $\xi \geq \xi_{2}$. Using (A4), (14) becomes

$$
\begin{aligned}
0 & \geq H^{\prime}(\xi)+G(a) H^{\prime}(\xi-\mu)+B_{1}(\xi)\left[G\left(v\left(\xi-\mu_{1}\right)\right)+G\left(a v\left(\xi-\mu-\mu_{1}\right)\right)\right] \\
& \geq H^{\prime}(\xi)+G(a) H^{\prime}(\xi-\mu)+\lambda B_{1}(\xi) G\left(h\left(\xi-\mu_{1}\right)\right)
\end{aligned}
$$

for $\xi \geq \xi_{3}>\xi_{2}+\mu_{1}$. Similarly, from the second equation of system (E), we get

$$
0 \geq \Delta H\left(\alpha_{i}\right)+G(a) \Delta H\left(\alpha_{i}-\mu\right)+\lambda B_{2}\left(\alpha_{i}\right) G\left(h\left(\alpha_{i}-\mu_{1}\right)\right)
$$


for $i \in \mathbb{N}$. Integrating (15) from $\xi_{3}$ to $+\infty$, we have

$$
\begin{aligned}
& \lambda \int_{\xi_{3}}^{\infty} B_{1}(\xi) G\left(h\left(\xi-\mu_{1}\right)\right) d \xi \\
& \quad \leq-[H(\xi)+G(a) H(\xi-\mu)]_{\xi_{3}}^{\infty}+\sum_{\xi_{3} \leq \alpha_{i}<\infty} \Delta\left[H\left(\alpha_{i}\right)+G(a) \Delta H\left(\alpha_{i}-\mu\right)\right] \\
& \quad \leq-[H(\xi)+G(a) H(\xi-\mu)]_{\xi_{3}}^{\infty}-\lambda \sum_{\xi_{3} \leq \alpha_{i}<\infty} B_{2}\left(\alpha_{i}\right) G\left(h\left(\alpha_{i}-\mu_{1}\right)\right) .
\end{aligned}
$$

Since $\lim _{\xi \rightarrow \infty} H(\xi)$ exists, then the above inequality becomes

$$
\lambda \int_{\xi_{3}}^{\infty} B_{1}(\xi) G\left(h\left(\xi-\mu_{1}\right)\right) d \xi+\lambda \sum_{\xi_{3} \leq \alpha_{i}<\infty} B_{2}\left(\alpha_{i}\right) G\left(h\left(\alpha_{i}-\mu_{1}\right)\right)<\infty .
$$

Consequently,

$$
\lambda\left[\int_{\xi_{3}}^{\infty} B_{1}(\xi) G\left(F^{+}\left(\xi-\mu_{1}\right)\right) d \xi+\sum_{\xi_{3} \leq \alpha_{i}<\infty} B_{2}\left(\alpha_{i}\right) G\left(F^{+}\left(\alpha_{i}-\mu_{1}\right)\right)\right]<\infty
$$

which contradicts (A5).

If $v(\xi)<0$ for $\xi \geq \xi_{0}$, then we set $v_{1}(\xi)=-v(\xi)$ for $\xi \geq \xi_{0}$ in (E), and we find

$$
\left\{\begin{array}{l}
\left(v_{1}(\xi)+b(\xi) v_{1}(\xi-\mu)\right)^{\prime}+b_{1}(\xi) G\left(v_{1}\left(\xi-\mu_{1}\right)\right)=\tilde{f}(\xi), \quad \xi \neq \mu_{k}, i \in \mathbb{N} \\
\triangle\left(v_{1}\left(\alpha_{i}\right)+b\left(\alpha_{i}\right) v_{1}\left(\alpha_{i}-\mu\right)\right)+b_{2}\left(\alpha_{i}\right) G\left(v_{1}\left(\alpha_{i}-\mu_{1}\right)\right)=\widetilde{g}\left(\alpha_{i}\right), \quad i \in \mathbb{N}
\end{array}\right.
$$

where $\tilde{f}(\xi)=-f(\xi), \widetilde{g}\left(\alpha_{i}\right)=-g\left(\alpha_{i}\right)$ because of (A4). Letting $\widetilde{F}(\xi)=-F(\xi)$, we have that

$$
-\infty<\liminf _{i \rightarrow \infty} \widetilde{F}(\xi)<0<\lim \sup _{i \rightarrow \infty} \widetilde{F}(\xi)<\infty
$$

and $\widetilde{F}^{\prime}(\xi)=\tilde{f}(\xi), \Delta \widetilde{F}\left(\alpha_{i}\right)=\widetilde{g}\left(\alpha_{i}\right)$ hold. Hence, proceeding as in the positive solution, we find a contradiction to (A6).

Thus, the theorem is proved.

Theorem 2.2 Under the assumptions $-1 \leq b(\xi) \leq 0$ for $\xi \in \mathbb{R}_{+}$, (A2)-(A4), and (A7)(A10), every solution of (E) oscillates.

Proof To prove by contradiction, we follow the proof of Theorem 2.1 to get that $H(\xi)$ is monotonic on $\left[\xi_{2}, \infty\right)$. So, we have the following two possible cases.

Case (i). Let $H(\xi)<0$ for $\xi \geq \xi_{2}$. Then, for $\xi \geq \xi_{2}$,

$$
-v(\xi-\mu) \leq b(\xi) v(\xi-\mu) \leq h(\xi)<F(\xi)
$$

we have $v\left(\xi-\mu_{1}\right)>-F\left(\xi+\mu-\mu_{1}\right), \xi \geq \xi_{3}>\xi_{2}$ and hence $v\left(\xi-\mu_{1}\right)>F^{-}\left(\xi+\mu-\mu_{1}\right)$, $\xi \geq \xi_{3}>\xi_{2}$. Thus (12) and (13) are reduced to

$$
\begin{aligned}
& H^{\prime}(\xi)+b_{1}(\xi) G\left(F^{-}\left(\xi+\mu-\mu_{1}\right)\right) \leq 0, \quad \xi \neq \alpha_{i}, i \in \mathbb{N}, \\
& \Delta H\left(\alpha_{i}\right)+b_{2}\left(\alpha_{i}\right) G\left(F^{-}\left(\alpha_{i}+\mu-\mu_{1}\right)\right) \leq 0, \quad i \in \mathbb{N},
\end{aligned}
$$


for $\xi \geq \xi_{4}$. Next, we are going to prove $-\infty<\lim _{\xi \rightarrow \infty} H(\xi)<0$. If not, letting $\lim _{\xi \rightarrow \infty} H(\xi)=\infty$ for $\xi \geq \xi_{4}$. For $0<\epsilon<\lambda-\gamma$, where $\lambda>\gamma>0$, there exists $\xi_{5}>\xi_{4}$ such that $F(\xi)<\gamma+\epsilon$ when $\xi \geq \xi_{5}$. Further, there is $\xi_{6}>\xi_{5}$ such that $\xi \geq \xi_{6}$ implies that $H(\xi)<-\lambda$, that is, $v(\xi) \leq v(\xi-\mu)-\lambda+\gamma+\epsilon$. For $\xi \geq \xi_{6}+j \mu$, we have $v(\xi) \leq \xi-j \mu+j(\gamma+\epsilon-\lambda)$. In particular, $v\left(\xi_{6}+j \mu\right) \leq v\left(\xi_{6}\right)+j(\gamma+\epsilon-\lambda)<0$ for large $\xi$, a contradiction to the fact $v(\xi)>0$ for $\xi \geq \xi_{0}$. Hence $-\infty<l<0$. Integrating (17) from $\xi_{6}$ to $+\infty$, we get

$$
\int_{\xi_{6}}^{\infty} b_{1}(\xi) G\left(F^{-}\left(\xi+\mu-\mu_{1}\right)\right) d \xi+\sum_{\xi_{6} \leq \alpha_{i}<\infty} b_{2}\left(\alpha_{i}\right) G\left(F^{-}\left(\alpha_{i}+\mu-\mu_{1}\right)\right)<\infty,
$$

which contradicts (A10).

Case (ii). Let $H(\xi)>0$ for $\xi \geq \xi_{2}$. We note that $v(\xi) \geq h(\xi)>F(\xi)$ for $\xi \geq \xi_{3}>\xi_{2}$. In this case, $\lim _{\xi \rightarrow \infty} H(\xi)$ exists. Because it happens that $v(\xi)>F^{+}(\xi)$ for $\xi \geq \xi_{3}$, then (12) and (13) can be viewed as

$$
\begin{aligned}
& H^{\prime}(\xi)+b_{1}(\xi) G\left(F^{+}\left(\xi-\mu_{1}\right)\right) \leq 0, \quad \xi \neq \alpha_{i}, i \in \mathbb{N} \\
& \Delta H\left(\alpha_{i}\right)+b_{2}\left(\alpha_{i}\right) G\left(F^{+}\left(\alpha_{i}-\mu_{1}\right)\right) \leq 0, \quad i \in \mathbb{N} .
\end{aligned}
$$

Integrating (18) from $\xi_{3}$ to $+\infty$, we have

$$
\int_{\xi_{3}}^{\infty} b_{1}(\xi) G\left(F^{+}\left(\xi-\mu_{1}\right)\right) d \xi+\sum_{\xi_{3} \leq \alpha_{i}<\infty} b_{2}\left(\alpha_{i}\right) G\left(F^{+}\left(\alpha_{i}-\mu_{1}\right)\right)<\infty,
$$

which is a contradiction to (A7).

The case $v(\xi)<0$ for $\xi \geq \xi_{0}$ is similar. Hence the details are omitted.

Thus, the theorem is proved.

Theorem 2.3 Under the assumption $-\infty<-b \leq b(\xi) \leq-1$ for $\xi \in \mathbb{R}_{+}$and $b>0$, and all the conditions of Theorem 2.2, every bounded solution of (E) oscillates.

Proof The proof of the theorem can be obtained from that of Theorem 2.2. Hence the details are omitted.

Remark 2.1 In Theorems 2.1-2.3, G could be linear, sublinear, or superlinear.

Remark 2.2 In Theorem 2.3, we are restricted on the solution of (E) to ensure the oscillation of (E). If we do not want to restrict on the solution, then $G$ should be superlinear. Hence, we have the following result.

Theorem 2.4 Under the assumptions $-\infty<-b \leq b(\xi) \leq-1$ for $\xi \in \mathbb{R}_{+}, b>0$, and $\mu \geq \mu_{1}$, and (A2), (A3), (A4), (A7), (A8), (A11)-(A13), every solution of (E) oscillates.

Proof The proof of the theorem follows from the proof of Theorem 2.2 except for the case when $H(\xi)<0, h(\xi)<0$ for $\xi \geq \xi_{3}$. Since $h(\xi) \geq b(\xi) v(\xi-\mu)$, then

$$
H(\xi)=h(\xi)-F(\xi) \geq b(\xi) v(\xi-\mu)-F(\xi) \quad \text { for } \xi \geq \xi_{3}
$$


implies that $H(\xi)-b(\xi) v(\xi-\mu) \geq-F(\xi)$ for $\xi \geq \xi_{3}$. Clearly, $H(\xi)-b(\xi) v(\xi-\mu)<0$ is not possible due to (A3) and the fact that $H(\xi)-b(\xi) v(\xi-\mu)=v(\xi)-F(\xi) \geq-F(\xi)$ if and only if $v(\xi)>0$ for $\xi \geq \xi_{3}$. Ultimately, $H(\xi)-b(\xi) v(\xi-\mu)>0$ and hence

$$
H(\xi)-b(\xi) v(\xi-\mu) \geq \max \{0,-F(\xi)\}=F^{-}(\xi),
$$

that is,

$$
H(\xi) \geq b(\xi) v(\xi-\mu)+F^{-}(\xi) \geq-b v(\xi-\mu)+F^{-}(\xi)>-b v(\xi-\mu)
$$

for $\xi \geq \xi_{4}>\xi_{3}$. Since $H(\xi)$ is decreasing and $\mu \geq \mu_{1}$, then it follows that

$$
-H(\xi) \leq-H\left(\xi+\mu-\mu_{1}\right)<b v\left(\xi-\mu_{1}\right) \quad \text { for } \xi \geq \xi_{4} .
$$

Therefore,

$$
\frac{G\left(v\left(\xi-\mu_{1}\right)\right)}{[-H(\xi)]^{\gamma}} \geq \frac{G\left(v\left(\xi-\mu_{1}\right)\right)}{b^{\gamma} v^{\gamma}\left(\xi-\mu_{1}\right)} \quad \text { for } \xi \geq \xi_{4} .
$$

Consequently,

$$
\begin{aligned}
-\frac{d}{d \xi}(-H(\xi))^{1-\gamma} & =-(1-\gamma)(-H(\xi))^{-\gamma}\left(-H^{\prime}(\xi)\right) \\
& =(\gamma-1)(-H(\xi))^{-\gamma} b_{1}(\xi) G\left(v\left(\xi-\mu_{1}\right)\right) \\
& \geq(\gamma-1) b_{1}(\xi) \frac{G\left(v\left(\xi-\mu_{1}\right)\right)}{b^{\gamma} v^{\gamma}\left(\xi-\mu_{1}\right)} \quad \text { for } \xi \geq \xi_{4}
\end{aligned}
$$

due to (12) and (20). We may note from (19) that $0>H(\xi)>-b v(\xi-\mu)+F^{-}(\xi)$ implies that $v\left(\xi-\mu_{1}\right)>b^{-1} F^{-}\left(\xi+\mu-\mu_{1}\right)$, and hence

$$
-\frac{d}{d \xi}(-H(\xi))^{1-\gamma} \geq(\gamma-1) b_{1}(\xi) \frac{G\left(b^{-1} F^{-}\left(\xi+\mu-\mu_{1}\right)\right)}{b^{\gamma}\left[b^{-1} F^{-}\left(\xi+\mu-\mu_{1}\right)\right]^{\gamma}}
$$

for $\xi \geq \xi_{4}$ due to (A11). Consequently,

$$
\begin{aligned}
& (\gamma-1) \int_{\xi_{4}}^{\xi} b_{1}(s) \frac{G\left(b^{-1} F^{-}\left(s+\mu-\mu_{1}\right)\right)}{\left[F^{-}\left(s+\mu-\mu_{1}\right)\right]^{\gamma}} d s \\
& \quad \leq-\left[-w^{1-\gamma}(s)\right]_{\xi_{4}}^{\xi}+\sum_{\xi_{4} \leq \alpha_{i}<\xi} \Delta\left[-w^{1-\gamma}\left(\alpha_{i}\right)\right] .
\end{aligned}
$$

Using the inequality

$$
\delta_{2}{ }^{1-\gamma}-\delta_{1}{ }^{1-\gamma} \leq(1-\gamma) b^{-\gamma}\left(\delta_{2}-\delta_{1}\right) \text { for } \delta_{1}<\delta_{2} \text { and } \gamma>1 \text {, }
$$

it follows that

$$
\begin{aligned}
\Delta\left[-H\left(\alpha_{i}\right)\right]^{1-\gamma} & =\left[-H\left(\alpha_{i}+0\right)\right]^{1-\gamma}-\left[-H\left(\alpha_{i}-0\right)\right]^{1-\gamma} \\
& \leq(1-\gamma)\left[-H\left(\alpha_{i}+0\right)\right]^{-\gamma} \Delta\left[-H\left(\alpha_{i}\right)\right]
\end{aligned}
$$




$$
\begin{aligned}
& =(1-\gamma) b_{2}\left(\alpha_{i}\right)\left[-H\left(\alpha_{i}+0\right)\right]^{-\gamma} G\left(v\left(\alpha_{i}-\mu_{1}\right)\right) \\
& =(1-\gamma) b_{2}\left(\alpha_{i}\right) \frac{G\left(v\left(\alpha_{i}-\mu_{1}\right)\right)}{\left[-H\left(\alpha_{i}\right)\right]^{\gamma}} \frac{\left[-H\left(\alpha_{i}\right)\right]^{\gamma}}{\left[-H\left(\alpha_{i}+0\right)\right]^{\gamma}} \\
& \leq(1-\gamma) b_{2}\left(\alpha_{i}\right) \frac{G\left(b^{-1} F^{-}\left(\alpha_{i}+\mu-\mu_{1}\right)\right)}{\left[F^{-}\left(\alpha_{i}+\mu-\mu_{1}\right)\right]^{\gamma}} \frac{\left[-H\left(\alpha_{i}\right)\right]^{\gamma}}{\left[-H\left(\alpha_{i}+0\right)\right]^{\gamma}}
\end{aligned}
$$

due to (13) and (20). From (13), it follows that $\Delta H\left(\alpha_{i}\right)=H\left(\alpha_{i}+0\right)-H\left(\alpha_{i}-0\right) \leq 0$, and hence

$$
q_{k}=\frac{\left[-H\left(\alpha_{i}-0\right)\right]}{\left[-H\left(\alpha_{i}+0\right)\right]}=\frac{\left[-H\left(\alpha_{i}\right)\right]}{\left[-H\left(\alpha_{i}+0\right)\right]} \leq 1, \quad i \in \mathbb{N},
$$

that is, $\left\{f_{k}\right\}$ is a bounded sequence. Let $c=\min _{i \in \mathbb{N}}\left\{q_{k}\right\}$. Then (22) becomes

$$
\Delta\left[-H\left(\alpha_{i}\right)\right]^{1-\gamma} \leq(1-\gamma) c^{\gamma} b_{2}\left(\alpha_{i}\right) \frac{G\left(b^{-1} F^{-}\left(\alpha_{i}+\mu-\mu_{1}\right)\right)}{\left[F^{-}\left(\alpha_{i}+\mu-\mu_{1}\right)\right]^{\gamma}} .
$$

Using (23) in (21), we obtain

$$
\begin{aligned}
& (\gamma-1)\left[\int_{\xi_{4}}^{\xi} b_{1}(s) \frac{G\left(b^{-1} F^{-}\left(s+\mu-\mu_{1}\right)\right)}{\left[F^{-}\left(s+\mu-\mu_{1}\right)\right]^{\gamma}} d s+c^{\gamma} \sum_{\xi_{4} \leq \alpha_{i}<\xi} b_{2}\left(\alpha_{i}\right) \frac{G\left(b^{-1} F^{-}\left(\alpha_{i}+\mu-\mu_{1}\right)\right)}{\left[F^{-}\left(\alpha_{i}+\mu-\mu_{1}\right)\right]^{\gamma}}\right] \\
& \quad \leq\left[w^{1-\gamma}(s)\right]_{\xi_{4}}^{\xi},
\end{aligned}
$$

that is,

$$
\begin{aligned}
& (\gamma-1) G\left(b^{-1}\right)\left[\int_{\xi_{4}}^{\xi} b_{1}(s) \frac{G\left(F^{-}\left(s+\mu-\mu_{1}\right)\right)}{\left[F^{-}\left(s+\mu-\mu_{1}\right)\right]^{\gamma}} d s+c^{\gamma} \sum_{\xi_{4} \leq \alpha_{i}<\xi} b_{2}\left(\alpha_{i}\right) \frac{G\left(F^{-}\left(\alpha_{i}+\mu-\mu_{1}\right)\right)}{\left[F^{-}\left(\alpha_{i}+\mu-\mu_{1}\right)\right]^{\gamma}}\right] \\
& \quad \leq\left[w^{1-\gamma}(s)\right]_{\xi_{4}}^{\xi}
\end{aligned}
$$

due to (A4). Taking limit as $\xi \rightarrow \infty$, we get a contradiction to (A13). This completes the proof of the theorem.

\section{Sufficient and necessary conditions for oscillation}

In this section, we are going to present the sufficient and necessary condition for oscillatory or asymptotic behavior of system (E).

Lemma $3.1([4])$ Considering $b, \xi, h \in C([0, \infty), \mathbb{R})$ such that $h(\xi)=v(\xi)+b(\xi) v(\xi-\mu)$, $\xi \geq \mu>0, v(\xi)>0$ for $\xi \geq \xi_{1}>\mu, \liminf _{\xi \rightarrow \infty} v(\xi)=0$ and $\lim _{\xi \rightarrow \infty} h(\xi)=L$ exist. If $b(\xi)$ satisfies one of the following conditions:

(i) $0 \leq a_{1} \leq b(\xi) \leq a_{2}<1$,

(ii) $1<a_{3} \leq b(\xi) \leq a_{4}<\infty$,

(iii) $-\infty<-a_{5} \leq b(\xi) \leq 0$,

where $a_{i}>0,1 \leq i \leq 5$, then $L=0$.

Theorem 3.1 Under the assumptions $0 \leq a_{1} \leq b(\xi) \leq a_{2}<1$ for $\xi \in \mathbb{R}_{+}$, (A1), and $G$ is Lipschitzian on $[c, d]$, where $0<c<d<\infty$, every solution of (E) either oscillates or $\lim _{\xi \rightarrow \infty} v(\xi)=0$ if and only if (A14) holds. 
Proof To prove sufficiency, we assume that (A14) holds and $v(\xi)$ is a solution of (E) on $\left[\xi_{v}, \infty\right]$ where $\xi_{v} \geq 0$. If $v(\xi)$ is oscillatory, then there is nothing to prove. Let the solution $v(\xi)>0$ for $\xi \geq \xi_{v}$. Then, proceeding as in Theorem 2.1, we have obtained (12) and (13) for $\xi \geq \xi_{1}>\xi_{0}+\mu_{1}$, where $\xi_{0}>\rho>\xi_{v}$. Hence, $H(\xi)$ is monotonic on $\left[\xi_{2}, \infty\right)$, where $\xi_{2}>\xi_{1}$. So, we have the following two possible cases.

Case (i). Let $H(\xi)>0$ for $\xi \geq \xi_{2}$. So, $\lim _{\xi \rightarrow \infty} H(\xi)$ exists and $\lim _{i \rightarrow \infty} H\left(\alpha_{i}\right)$ exists. Now, we are going to prove that $v(\xi)$ is bounded. If not, there exists $\left\{\eta_{n}\right\}$ such that $\eta_{n} \rightarrow \infty$ as $n \rightarrow \infty, v\left(\eta_{n}\right) \rightarrow \infty$ as $n \rightarrow \infty$ and

$$
v\left(\eta_{n}\right)=\max \left\{v(s): \xi_{2} \leq s \leq \eta_{n}\right\}
$$

Therefore,

$$
H\left(\eta_{n}\right)=v\left(\eta_{n}\right)+b\left(\eta_{n}\right) v\left(\eta_{n}-\mu\right)-F\left(\eta_{n}\right) \geq\left(1+a_{1}\right) v\left(\eta_{n}\right)-F\left(\eta_{n}\right) \rightarrow+\infty, \quad \text { as } n \rightarrow \infty,
$$

which is a contradiction. So, $v(\xi)$ is bounded. The same contradiction holds when $H(\xi)<0$ for $\xi \geq \xi_{2}$. Consequently, $H(\xi)$ is bounded and $\lim _{\xi \rightarrow \infty} H(\xi)$ exists. Our aim is to show that $\lim _{\xi \rightarrow \infty} v(\xi)=0$. For this, we need to show that $\liminf _{\xi \rightarrow \infty} v(\xi)=0$ and $\lim _{\sup _{\xi \rightarrow \infty}} v(\xi)=$ 0 for every $\xi$ and $\alpha_{i}$. First, we are going to prove $\liminf _{\xi \rightarrow \infty} v(\xi)=0$. To prove this by contradiction, letting $\liminf _{\xi \rightarrow \infty} v(\xi) \neq 0$, then for $\xi_{3}>\xi_{2}$ and $\gamma>0$, we have $v\left(\xi-\mu_{1}\right) \geq$ $\gamma>0$ for $\xi \geq \xi_{3}$. Ultimately,

$$
\begin{aligned}
& \int_{\xi_{3}}^{\xi} b_{1}(\kappa) G\left(v\left(\kappa-\mu_{1}\right)\right) d \kappa+\sum_{\xi_{3} \leq \alpha_{i}<\xi} b_{2}\left(\alpha_{i}\right) G\left(v\left(\alpha_{i}-\mu_{1}\right)\right) \\
& \geq G(\gamma)\left[\int_{\xi_{3}}^{\xi} b_{1}(\kappa) d \kappa+\sum_{\xi_{3} \leq \alpha_{i}<\xi} b_{2}\left(\alpha_{i}\right)\right] \rightarrow+\infty, \quad \text { as } \xi \rightarrow \infty,
\end{aligned}
$$

due to (A14). Again, if we integrate (12) from $\xi_{3}$ to $\xi$, we get

$$
[H(s)]_{\xi_{3}}^{\xi}+\int_{\xi_{3}}^{\xi} b_{1}(\kappa) G\left(v\left(s-\mu_{1}\right)\right) d s-\sum_{\xi_{3} \leq \alpha_{i}<\xi} \Delta H\left(\alpha_{i}\right)=0,
$$

and hence, using (13), it follows that

$$
\begin{aligned}
& \int_{\xi_{3}}^{\xi} b_{1}(\kappa) G\left(v\left(\kappa-\mu_{1}\right)\right) d \kappa+\sum_{\xi_{3} \leq \alpha_{i}<\xi} b_{2}\left(\alpha_{i}\right) G\left(v\left(\alpha_{i}-\mu_{1}\right)\right) \\
& =-[H(\kappa)]_{\xi_{3}}^{\xi}<\infty, \quad \text { as } \xi \rightarrow \infty .
\end{aligned}
$$

Using (24) and (25), we have a contradiction. So, $\liminf _{\xi \rightarrow \infty} v(\xi)=0$ for $\xi \geq \xi_{3}$. Since $\lim _{\xi \rightarrow \infty} H(\xi)$ exists, then $\lim _{\xi \rightarrow \infty} h(\xi)$ exists due to (A1). Therefore, by Lemma 3.1, we conclude that $\lim _{\xi \rightarrow \infty} h(\xi)=0$. Consequently,

$$
0=\lim _{\xi \rightarrow \infty} h(\xi)=\lim \sup _{\xi \rightarrow \infty}(v(\xi)+b(\xi) v(\xi-\mu)) \geq \lim \sup _{\xi \rightarrow \infty} v(\xi)
$$


implies that $\lim \sup _{\xi \rightarrow \infty} v(\xi)=0$. Ultimately, $\lim _{\xi \rightarrow \infty} v(\xi)=0$ for $\xi \neq \alpha_{i}$, where $i \in \mathbb{N}$. Note that $\left\{v\left(\alpha_{i}-0\right)\right\}$ and $\left\{v\left(\alpha_{i}+0\right)\right\}$ are sequences of real numbers and $v$ is continuous. So, we have $\lim _{i \rightarrow \infty} v\left(\alpha_{i}-0\right)=0$ and $\lim _{i \rightarrow \infty} v\left(\alpha_{i}+0\right)=0$ due to $\liminf _{\xi \rightarrow \infty} v(\xi)=0$ and $\lim \sup _{\xi \rightarrow \infty} v(\xi)=0$ respectively. Hence, for all $\xi$ and $\alpha_{i}$, where $i \in \mathbb{N}$, we have $\lim _{\xi \rightarrow \infty} v(\xi)=0$.

The similar procedure can be followed when $v(\xi)<0$ for $\xi \geq \xi_{v}$ to show that $\lim _{\xi \rightarrow \infty} v(\xi)=0$.

Next, to prove the necessary part, we assume that

$$
\int_{0}^{\infty} b_{1}(\xi) d \xi+\sum_{i=1}^{\infty} b_{2}\left(\alpha_{i}\right)<\infty
$$

and we must have to prove that the impulsive system (E) has a non-oscillatory solution and $\lim _{\xi \rightarrow \infty} v(\xi) \neq 0$. If possible, let there exist $\xi_{1}>0$ such that

$$
\int_{\xi_{1}}^{\infty} b_{1}(\kappa) d \kappa+\sum_{i=1}^{\infty} b_{2}\left(\alpha_{i}\right)<\frac{1-a_{2}}{5 L}
$$

where $L=\max \left\{L_{1}, G(1)\right\}$ and $L_{1}$ is the Lipschitz constant on $\left[\frac{1-a_{2}}{10}, 1\right]$. By $\left(A_{1}\right)$, let $\lim _{\xi \rightarrow \infty} F(\xi)=M$. Then we can find $\xi_{2}>\xi_{1}$ so that $|F(\xi)-M|<\frac{1-a_{2}}{10}$ for $\xi \geq \xi_{2}$. For $\xi_{3}>\max \left\{\xi_{1}, \xi_{2}\right\}$, we assume $X=\mathrm{BC}\left(\left[\xi_{3}, \infty\right), \mathbb{R}\right)$, (that is, the space bounded continuous real-valued functions on $\left.\left[\xi_{3}, \infty\right)\right)$. Therefore, $X$ is a Banach space with respect to sup norm defined by

$$
\|x\|=\sup \left\{\left|v_{1}(\xi)\right|: \xi \geq \xi_{3}\right\}
$$

Let us define

$$
S=\left\{v \in X: \frac{1-a_{2}}{10} \leq v(\xi) \leq 1, \xi \geq \xi_{3}\right\} .
$$

Clearly, $S$ is a convex and closed subspace of $X$. Let $\Omega: S \rightarrow S$ be an operator defined by

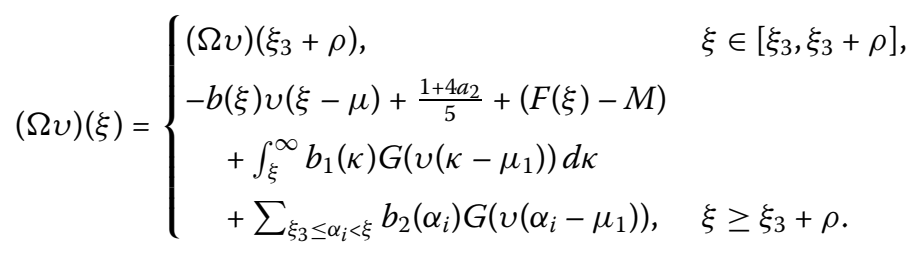

For every $\xi \in S$,

$$
\begin{aligned}
(\Omega v)(\xi) & \leq \frac{1-a_{2}}{10}+\frac{1+4 a_{2}}{5}+G(1)\left[\int_{\xi}^{\infty} b_{1}(\kappa) d \kappa+\sum_{\xi_{3} \leq \alpha_{i}<\xi} b_{2}\left(\alpha_{i}\right)\right] \\
& <\frac{1-a_{2}}{10}+\frac{1+4 a_{2}}{5}+\frac{1-a_{2}}{5}=\frac{1+a_{2}}{2}<1
\end{aligned}
$$


and

$$
\begin{aligned}
(\Omega v)(\xi) & \geq-b(\xi) v(\xi-\mu)+\frac{1+4 a_{2}}{5}+(F(\xi)-M) \\
& \geq-a_{2}+\frac{1+4 a_{2}}{5}-\frac{1-a_{2}}{10}=\frac{1-a_{2}}{10}
\end{aligned}
$$

imply that $(\Omega v) \in S$. Again, for $v_{1}, v_{2} \in S$,

$$
\begin{aligned}
& \left|\left(\Omega v_{1}\right)(\xi)-\left(\Omega v_{2}\right)(\xi)\right| \\
& \quad \leq|b(\xi)|\left|v_{1}(\xi-\mu)-v_{2}(\xi-\mu)\right|+L_{1} \int_{\xi}^{\infty} b_{1}(\kappa)\left|v_{1}\left(\kappa-\mu_{1}\right)-v_{2}\left(\kappa-\mu_{1}\right)\right| d \kappa \\
& \quad+L_{1} \sum_{\xi_{3} \leq \alpha_{i}<\xi} b_{2}\left(\alpha_{i}\right)\left|v_{1}\left(\alpha_{i}-\mu_{1}\right)-v_{2}\left(\alpha_{i}-\mu_{1}\right)\right|,
\end{aligned}
$$

that is,

$$
\begin{aligned}
\left|\left(\Omega v_{1}\right)(\xi)-\left(\Omega v_{2}\right)(\xi)\right| & \leq a_{2}\left\|v_{1}-v_{2}\right\|+L_{1}\left\|v_{1}-v_{2}\right\|\left[\int_{\xi}^{\infty} b_{1}(\kappa) d \kappa+\sum_{\xi_{3} \leq \alpha_{i}<\xi} b_{2}\left(\alpha_{i}\right)\right] \\
& <\left(a_{2}+\frac{1-a_{2}}{5}\right)\left\|v_{1}-v_{2}\right\|,
\end{aligned}
$$

that is, $\Omega$ is a contraction mapping with the contraction $\left(a_{2}+\frac{1-a_{2}}{5}\right)=\frac{1+4 a_{2}}{5}<1$. Note that $\Omega$ is a contraction on $S$ and $S$ is complete. Then, by using the Banach fixed point theorem, $\xi$ has a unique fixed point on $\left[\frac{1-a_{2}}{10}, 1\right]$. Hence $\Omega v=v$ and

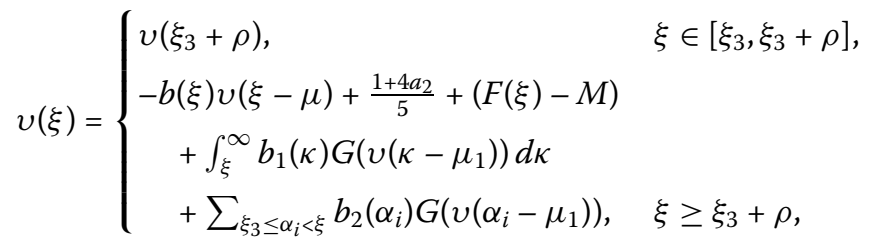

is a non-oscillatory solution of system (E) on $\left[\frac{1-a_{2}}{10}, 1\right]$ such that $\lim _{\xi \rightarrow \infty} v(\xi) \neq 0$.

Thus, the theorem is proved.

Theorem 3.2 Under assumptions $1<a_{3} \leq b(\xi) \leq a_{4}<\infty$ for $\xi \in \mathbb{R}_{+}, a_{3}^{2}>a_{4}$, (A1), and $G$ is Lipschitzian on $[c, d]$, where $0<c<d<\infty$, every solution of system (E) either oscillates or $\lim _{\xi \rightarrow \infty} v(\xi)=0$ if and only if (A14) holds.

Proof The proof of the sufficient part is the same as in the proof of Theorem 3.1.

To prove necessity, we assume that (26) holds. So, $\xi_{1}>0$ we have

$$
\int_{\xi_{1}}^{\infty} b_{1}(\xi) d \xi+\sum_{i=1}^{\infty} b_{2}\left(\alpha_{i}\right)<\frac{a_{3}-1}{2 L}
$$

where $L=\max \left\{L_{1}, L_{2}\right\}$ and $L_{1}$ is the Lipschitz constant of $G$ on $[c, d]$, where $L_{2}=G(d)$ such that

$$
a=\frac{2 c\left(a_{3}^{2}-a_{4}\right)-a_{4}\left(a_{3}+a_{3}^{2}-2\right)}{2 a_{3}^{2} a_{4}}, \quad b=\frac{a_{3}-1+c}{a_{3}}, \quad c>\frac{a_{4}\left(a_{3}+a_{3}^{2}-2\right)}{2\left(a_{3}^{2}-a_{4}\right)}>0 .
$$


Also, we can find $\xi_{2}>0$ such that $|F(\xi)-M|<\frac{1}{2}\left(a_{3}-1\right)$ for $\xi \geq \xi_{2}>\xi_{1}$. Next, we define a Banach space $X$ as in the proof of Theorem 3.1 with respect to the sup norm

$$
\|x\|=\sup \left\{\left|v_{1}(\xi)\right|: \xi \geq \xi_{2}\right\}
$$

Define

$$
S=\left\{v \in X: a \leq v(\xi) \leq b, \xi \geq \xi_{2}\right\}
$$

Clearly, $S$ is a convex and closed subspace of $X$. Let $\Omega: S \rightarrow S$ be an operator defined by

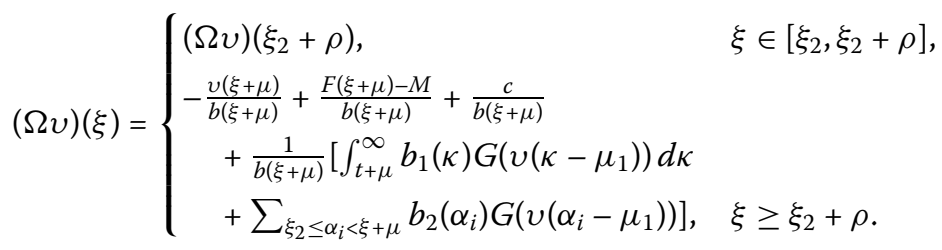

For every $v \in S$,

$$
\begin{aligned}
(\Omega v)(\xi) & \leq \frac{G(d)}{b(\xi+\mu)}\left[\int_{\xi+\mu}^{\infty} b_{1}(\kappa) d \kappa+\sum_{\xi_{2} \leq \alpha_{i}<\xi+\mu} b_{2}\left(\alpha_{i}\right)\right]+\frac{a_{3}-1}{2 b(\xi+\mu)}+\frac{c}{b(\xi+\mu)} \\
& \leq \frac{1}{a_{3}}\left[\frac{2\left(a_{3}-1\right)}{2}+c\right]=b
\end{aligned}
$$

and

$$
\begin{aligned}
(\Omega v)(\xi) & \geq-\frac{v(\xi+\mu)}{b(\xi+\mu)}+\frac{F(\xi+\mu)-M}{b(\xi+\mu)}+\frac{c}{b(\xi+\mu)}>-\frac{b}{a_{3}}-\frac{a_{3}-1}{2 a_{3}}+\frac{c}{a_{4}} \\
& =-\frac{a_{3}-1+c}{a_{3}^{2}}-\frac{a_{3}-1}{2 a_{3}}+\frac{c}{a_{4}}=\frac{2 c\left(a_{3}^{2}-a_{4}\right)-a_{4}\left(a_{3}-2+a_{3}^{2}\right)}{2 a_{3}^{2} a_{4}}=a
\end{aligned}
$$

implies that $\Omega \in S$. For $v_{1}, v_{2} \in S$,

$$
\begin{aligned}
\left|\left(\Omega v_{1}\right)(\xi)-\left(\Omega v_{2}\right)(\xi)\right| \leq & \frac{1}{|b(\xi+\mu)|}\left|v_{1}(\xi+\mu)-v_{2}(\xi+\mu)\right| \\
& +\frac{G(b)}{|b(\xi+\mu)|}\left[\int_{\xi+\mu}^{\infty} b_{1}(\kappa)\left|v_{1}\left(\kappa-\mu_{1}\right)-v_{2}\left(\kappa-\mu_{1}\right)\right| d \kappa\right. \\
& \left.+\sum_{\xi_{2} \leq \alpha_{i}<\xi+\mu} b_{2}\left(\alpha_{i}\right)\left|v_{1}\left(\alpha_{i}-\mu_{1}\right)-v_{2}\left(\alpha_{i}-\mu_{1}\right)\right|\right],
\end{aligned}
$$

that is,

$$
\begin{aligned}
& \left|\left(\Omega v_{1}\right)(\xi)-\left(\Omega v_{2}\right)(\xi)\right| \\
& \quad \leq \frac{1}{a_{3}}\left\|v_{1}-v_{2}\right\|+\frac{G(b)}{a_{3}}\left\|v_{1}-v_{2}\right\|\left[\int_{\xi+\mu}^{\infty} b_{1}(\kappa) d \kappa+\sum_{\xi_{2} \leq \alpha_{i}<\xi+\mu} b_{2}\left(\alpha_{i}\right)\right] \\
& \quad<\left(\frac{1}{a_{3}}+\frac{a_{3}-1}{2 a_{3}}\right)\left\|v_{1}-v_{2}\right\|,
\end{aligned}
$$


that is, $\Omega$ is a contraction mapping with the contraction $\left(\frac{1}{a_{3}}+\frac{a_{3}-1}{2 a_{3}}\right)<1$. Hence, by the Banach fixed point theorem, $\Omega$ has a unique fixed point on $[a, b]$ which is a non-oscillatory (specially positive) solution of system (E).

Thus, the theorem is proved.

Theorem 3.3 Under the assumptions $-1<-a_{5} \leq b(\xi) \leq 0$ for $\xi \in \mathbb{R}_{+}, a_{5}>0$ and (A1), every solution of system (E) either oscillates or $\lim _{\xi \rightarrow \infty} v(\xi)=0$ if and only if (A14) holds.

Proof For the sufficient part, we follow the proof of Theorem 3.1 to show that $v(\xi)$ is bounded when $H(\xi)>0$ for $\xi \geq \xi_{2}$. Also, $v(\xi)$ is bounded when $H(\xi)<0$ for $\xi \geq \xi_{2}$. Consequently, $\lim _{\xi \rightarrow \infty} H(\xi)$ exists and hence $\lim _{\xi \rightarrow \infty} h(\xi)$ exists. By Theorem 3.1, it is easy to prove that $\liminf _{\xi \rightarrow \infty} v(\xi)=0$, and by Lemma 3.1, $\lim _{\xi \rightarrow \infty} h(\xi)=0$. So,

$$
\begin{aligned}
0 & =\lim _{\xi \rightarrow \infty} h(\xi)=\lim \sup _{\xi \rightarrow \infty}(v(\xi)+b(\xi) v(\xi-\mu)) \\
& \geq \lim \sup _{\xi \rightarrow \infty} v(\xi)+\liminf _{\xi \rightarrow \infty}\left(-a_{5} v(\xi-\mu)\right) \\
& =\left(1-a_{5}\right) \lim \sup _{\xi \rightarrow \infty} v(\xi)
\end{aligned}
$$

implies that $\limsup _{\xi \rightarrow \infty} v(\xi)=0$. The rest of the sufficient part comes from the proof of Theorem 3.1.

Next, we suppose that (26) holds. Then there exist $\xi_{1}, \xi_{2}>0$ such that

$$
\int_{\xi_{1}}^{\infty} b_{1}(\xi) d \xi+\sum_{i=1}^{\infty} b_{2}\left(\alpha_{i}\right)<\frac{1-a_{5}}{10 G(1)} \quad \text { for } \xi \geq \xi_{1}
$$

and $|F(\xi)-M|<\frac{1-a_{5}}{20}$ for $\xi \geq \xi_{2}$. Next, we define a Banach space $X$ as in the proof of Theorem 3.1 with respect to the sup norm

$$
\|x\|=\sup \left\{\left|v_{1}(\xi)\right|: \xi \geq \xi_{2}\right\}
$$

Let $K=\left\{x \in X: v_{1}(\xi) \geq 0, \xi \geq \xi_{3}\right\}$. Then $X$ is a partially ordered Banach space (see, for instance, [54], p. 30). For $x, y \in X$, we define $x \leq y$ if and only if $x-y \in K$. Let

$$
S=\left\{v \in X: \frac{1-a_{5}}{20} \leq v(\xi) \leq 1, \xi \geq \xi_{3}\right\}
$$

If $v_{0}(\xi)=\frac{1-a_{5}}{20}$, then $v_{0} \in S$ and $v_{0}=$ g.l.b $S$. Further, if $\Phi \subset S^{*} \subset S$, then

$$
S^{*}=\left\{v \in X: l_{1} \leq v(\xi) \leq l_{2}, \frac{1-a_{5}}{20} \leq l_{1}, l_{2} \leq 1\right\}
$$


Let $v_{0}(\xi)=l_{2}^{\prime}, \xi \geq \xi_{3}$, where $l_{2}^{\prime}=\sup \left\{l_{2}: \frac{1-a_{5}}{20} \leq l_{2} \leq 1\right\}$. Then $v_{0} \in S$ and $v_{0}=1$.u.b $S^{*}$. For $\xi_{4}=\xi_{3}+\rho$, define $\xi: S \rightarrow S$ by

$$
(\Omega v)(\xi)=\left\{\begin{array}{rrr}
(\Omega v)\left(\xi_{4}\right), & \xi \in\left[\xi_{3}, \xi_{4}\right], \\
-b(\xi) v(\xi-\mu)+\frac{1-a_{5}}{10}+(F(\xi)-M) & \\
& \quad+\int_{\xi}^{\infty} b_{1}(\kappa) G\left(v\left(\kappa-\mu_{1}\right)\right) d \kappa & \\
& +\sum_{\xi_{4} \leq \alpha_{i}<\xi} b_{2}\left(\alpha_{i}\right) G\left(v\left(\alpha_{i}-\mu_{1}\right)\right), & \xi \geq \xi_{4} .
\end{array}\right.
$$

For every $v \in S$,

$$
\begin{aligned}
(\Omega v)(\xi) & \leq a_{5}+G(1)\left[\int_{\xi}^{\infty} b_{1}(\kappa) d \kappa+\sum_{\xi_{4} \leq \alpha_{i}<\xi} b_{2}\left(\alpha_{i}\right)\right]+\frac{1-a_{5}}{20}+\frac{1-a_{5}}{10} \\
& <\frac{1+3 a_{5}}{4}<1
\end{aligned}
$$

and

$$
(\Omega v)(\xi) \geq \frac{1-a_{5}}{10}+(F(\xi)-M) \frac{1-a_{5}}{10}-\frac{1-a_{5}}{20}=\frac{1-a_{5}}{20}
$$

implies that $\Omega v \in S$. Now, for $v_{1}, v_{2} \in S$, it is easy to verify that $v_{1} \leq v_{2}$ implies that $\left(\Omega v_{1}\right) \leq$ $\left(\Omega v_{2}\right)$. Hence, by the Knaster-Tarski fixed point theorem (see, e.g., [54], Theorem 1.7.3), $\Omega$ has a unique fixed point such that $\lim _{\xi \rightarrow \infty} v(\xi) \neq 0$.

This completes the proof of the theorem.

Theorem 3.4 Under the assumptions $-\infty<-a_{5} \leq b(\xi) \leq-a_{6}<-1$ for $\xi \in \mathbb{R}_{+}, a_{5}, a_{6}>0$, (A1), and $G$ is Lipschitzian on the intervals of the form $[c, d]$, where $0<c<d<\infty$, every bounded solution of (E) either oscillates or $\lim _{\xi \rightarrow \infty} v(\xi)=0$ if and only if (A14) holds.

Proof The proof is totally the same as in the proof of Theorem 3.2, but, for the necessary part, we provide the following settings:

$$
\int_{\xi_{1}}^{\infty} b_{1}(\kappa) d \kappa+\sum_{i=1}^{\infty} b_{2}\left(\alpha_{i}\right)<\frac{a_{6}-1}{2 L} \quad \text { and } \quad|F(\xi)-M|>\frac{1}{2}\left(a_{6}-1\right)
$$

where $L=\max \left\{L_{1}, L_{2}\right\}$ and $L_{1}$ is the Lipschitz constant of $G$ on $[c, d]$, where $L_{2}=G(d)$ such that

$$
\begin{aligned}
& a=\frac{c a_{6}-a_{5}\left(a_{6}-1\right)}{a_{5} a_{6}}, \\
& b=\frac{1}{2}+\frac{c}{a_{6}-1}, \\
& c>\frac{a_{5}\left(a_{6}-1\right)}{a_{6}}>0,
\end{aligned}
$$


and

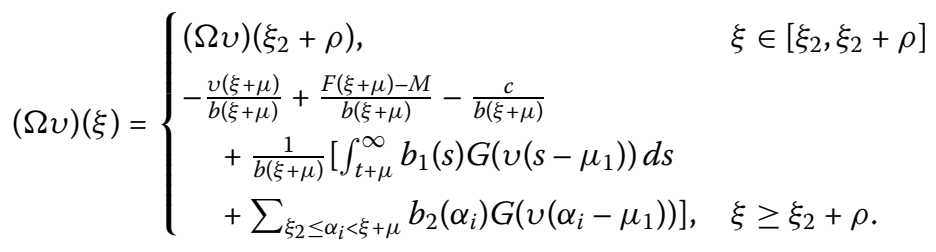

This completes the proof of the theorem.

Remark 3.1 In Theorems 3.1-3.4, we do not have any restriction on $G$ (that is, $G$ could be linear, sublinear, or superlinear).

\section{Conclusion}

In [20], the author studied the oscillatory behavior of solutions of the impulsive system

$$
\begin{cases}(v(\xi)+b(\xi) v(\xi-\mu))^{\prime}+b_{1}(\xi) G\left(v\left(\xi-\mu_{1}\right)\right)=0, & \xi \neq \alpha_{i}, i \in \mathbb{N} \\ \triangle\left(v\left(\alpha_{i}\right)+B_{2}\left(\alpha_{i}\right) v\left(\alpha_{i}-\mu\right)\right)+q_{k} G\left(v\left(\alpha_{i}-\mu_{1}\right)\right)=0, & i \in \mathbb{N}\end{cases}
$$

under the sufficient condition

$$
\int_{0}^{\infty} b_{1}(\xi) d \xi+\sum_{i=1}^{\infty} b_{1}\left(\alpha_{i}\right)=\infty
$$

Because of Theorem 3.1 [20], (27) could be a sufficient and necessary condition for the oscillatory and asymptotic behavior of solutions of system (E1) for different ranges of the neutral coefficient $b(\xi)$. We guess that (27) could be a sufficient and necessary condition for the oscillation of a non-homogeneous counterpart of (E). In this work, we have obtained sufficient conditions for the oscillation of (E), which is presented in Sect. 2, and in Sect. 3 we have established sufficient and necessary conditions for the oscillatory or asymptotic behavior of (E).

It would be of interest to examine the oscillation of (E) with a different neutral coefficient; see, e.g., the papers [43, 46, 47, 50-53] for more details. Furthermore, it is also interesting to analyze the oscillation of (E) with a nonlinear neutral term; see, e.g., the paper [49] for more details.

Remark 4.1 Theorems 3.1-3.4 hold true for $M=0$.

Remark 4.2 Lemma 3.1 does not hold when $b(\xi) \equiv 1$ for all $\xi$ (see, e.g., [54]), and the present study does not allow us when $b(\xi) \equiv-1$ for all $\xi$. Thus, in this paper, we have obtained necessary and sufficient conditions for the oscillatory or asymptotic behavior of (E) except $b(\xi)= \pm 1$ for all $\xi$. Hence, it is clear that a different method is necessary to study the oscillatory or asymptotic behavior of $(\mathrm{E})$ when $b(\xi)= \pm 1$. However, we have established sufficient conditions for $b(\xi)= \pm 1$ in Sect. 2 .

\section{Examples}

In this section, we provide two examples to validate our main results. 
Example 5.1 Consider the impulsive system

$$
\left\{\begin{array}{l}
(v(\xi)+2 v(\xi-1))^{\prime}+4(\xi-1)^{3} v^{3}(\xi-1)=-\frac{2}{\xi^{3}}, \quad \xi>1 \\
\Delta\left(v\left(\alpha_{i}\right)+2 v\left(\alpha_{i}-1\right)\right)+b_{2}\left(\alpha_{i}\right) v^{3}\left(\alpha_{i}-1\right)=-\frac{4 h 2^{i}}{\left(4^{i}-h^{2}\right)^{2}}
\end{array}\right.
$$

where $b_{2}\left(\alpha_{i}\right)=\frac{8 b_{2}\left(2^{i}-1\right)\left(2^{i}-1-h\right)^{6}}{\left(\left(2^{i}-1\right)^{2}-h^{2}\right)^{2}}, \alpha_{i}=2^{i}, i \in \mathbb{N}$, and $G(\xi)=\xi^{3}$. If we choose $F(\xi)=\frac{1}{\xi^{2}}$, then $F^{\prime}(\xi)=-\frac{2}{\xi^{3}}$ and

$$
\begin{aligned}
\Delta F\left(\alpha_{i}\right) & =F\left(\alpha_{i}+h\right)-F\left(\alpha_{i}-h\right) \\
& =F\left(2^{i}+h\right)-F\left(2^{i}-h\right) \\
& =-\frac{4 h 2^{i}}{\left(4^{i}-h^{2}\right)^{2}}=g\left(\alpha_{i}\right), \quad i \in \mathbb{N} .
\end{aligned}
$$

Clearly, (A14) holds. Since the conditions of Theorem 3.2 are true for $\left(E_{1}\right)$, then every solution of $\left(E_{1}\right)$ either oscillates or tends to zero as $\xi \rightarrow \infty$. In particular, $v(\xi)=\frac{1}{\xi^{2}}$ is a solution of the impulsive system $\left(E_{1}\right)$.

Example 5.2 Consider the impulsive system

$$
\left\{\begin{array}{l}
(v(\xi)+v(\xi-\pi))^{\prime}+v\left(\xi-\frac{\pi}{4}\right)=\cos \left(\xi-\frac{\pi}{4}\right), \quad \xi>\frac{\pi}{4}, \\
\Delta\left(v\left(\alpha_{i}\right)+v\left(\alpha_{i}-\pi\right)\right)+b_{2}\left(\alpha_{i}\right) y\left(\alpha_{i}-\frac{\pi}{4}\right)=2 \sin (h) \cos \left(k-\frac{\pi}{4}\right)
\end{array}\right.
$$

where $b_{2}\left(\alpha_{i}\right)=\frac{2}{1+\cot (h)}, \alpha_{i}=i, i \in \mathbb{N}, G(\xi)=\xi, f(\xi)=\cos \left(\xi-\frac{\pi}{4}\right)$. Indeed, if we choose $F(\xi)=$ $\sin \left(\xi-\frac{\pi}{4}\right)$, then $F^{\prime}(\xi)=f(\xi)$ and

$$
\begin{aligned}
\Delta F\left(\alpha_{i}\right) & =F\left(\alpha_{i}+h\right)-F\left(\alpha_{i}-h\right) \\
& =F(i+h)-F(i-h) \\
& =\sqrt{2} \sin (h)(\sin (i)+\cos (i))=g\left(\alpha_{i}\right), \quad i \in \mathbb{N} .
\end{aligned}
$$

Clearly,

$$
F^{+}(\xi)= \begin{cases}\sin \left(\xi-\frac{\pi}{4}\right), & 2 n \pi+\frac{\pi}{4} \leq \xi \leq 2 n \pi+\frac{5 \pi}{4} \\ 0, & \text { otherwise }\end{cases}
$$

and

$$
F^{-}(\xi)= \begin{cases}-\sin \left(\xi-\frac{\pi}{4}\right), & 2 n \pi+\frac{5 \pi}{4} \leq \xi \leq 2 n \pi+\frac{9 \pi}{4} \\ 0, & \text { otherwise }\end{cases}
$$

imply that

$$
F^{+}\left(\xi-\frac{\pi}{4}\right)= \begin{cases}-\cos (\xi), & 2 n \pi+\frac{\pi}{2} \leq \xi \leq 2 n \pi+\frac{3 \pi}{2} \\ 0, & \text { otherwise }\end{cases}
$$


and

$$
F^{-}\left(\xi-\frac{\pi}{2}\right)= \begin{cases}\sin (\xi), & 2 n \pi+\frac{3 \pi}{2} \leq \xi \leq 2 n \pi+\frac{5 \pi}{2} \\ 0, & \text { otherwise. }\end{cases}
$$

Since

$$
\int_{\frac{\pi}{2}}^{\infty} F^{+}\left(\xi-\frac{\pi}{4}\right) d \xi=\sum_{n=0}^{\infty} \int_{2 n \pi+\frac{\pi}{2}}^{2 n \pi+\frac{3 \pi}{2}}[-\cos (\xi)] d \xi=\infty
$$

then, for $n=0,1,2, \ldots$, we get

$$
\int_{\frac{\pi}{2}}^{\infty} F^{+}\left(\xi-\frac{\pi}{4}\right) d \xi+\sum_{i=1}^{\infty}\left(\frac{2}{1+\cot (h)}\right) F^{+}\left(i-\frac{\pi}{4}\right)=\infty
$$

Clearly, (A2)-(A6) are satisfied. Hence, by Theorem 2.1, every solution of $\left(E_{2}\right)$ is oscillatory. In particular, $v_{1}(\xi)=\cos (\xi)$ is a solution of $\left(E_{2}\right)$.

\section{Acknowledgements}

The authors extend their thanks to the Deanship of Scientific Research at King Khalid University for funding this work through the small research groups under grant number RGP. 1/372/42. The authors thank the editors and the reviewers for their useful comments.

\section{Funding}

This research work was supported by the Deanship of Scientific Research at King Khalid University under Grant number RGP. 1/372/42

\section{Availability of data and materials}

Not applicable.

\section{Competing interests}

The authors declare that they have no competing interests.

\section{Authors' contributions}

The authors declare that they have read and approved the final manuscript.

\section{Author details}

${ }^{1}$ Department of Mathematics, JIS College of Engineering, Kalyani 741235, India. ${ }^{2}$ Department of Mathematics and Computer Science, Faculty of Arts and Sciences, Çankaya University Ankara, 06790 Etimesgut, Turkey. ${ }^{3}$ Instiute of Space Sciences, Magurele-Bucharest, 077125 Magurele, Romania. ${ }^{4}$ Department of Medical Research, China Medical University Hospital, China Medical University, Taichung, 40402, Taiwan, Republic of China. ${ }^{5}$ Department of Civil Engineering, College of Engineering, King Khalid University, Abha 61421, Saudi Arabia. ${ }^{6}$ Department of Civil Engineering, High Institute of Technological Studies, Mrezgua University Campus, Nabeul 8000, Tunisia. ${ }^{7}$ Department of Mathematics, Faculty of Science, Mansoura University, Mansoura 35516, Egypt.

\section{Publisher's Note}

Springer Nature remains neutral with regard to jurisdictional claims in published maps and institutional affiliations.

Received: 14 April 2021 Accepted: 1 June 2021 Published online: 09 June 2021

\section{References}

1. Bainov, D.D., Simeonov, P.S.: Impulsive Differential Equations: Asymptotic Properties of the Solutions. Series on Advances in Mathematics for Applied Sciences, vol. 28. World Scientific, Singapore (1995)

2. Lakshmikantham, V., Bainov, D.D., Simeonov, P.S.: Oscillation Theory of Impulsive Differential Equations. World Scientific, Singapore (1989)

3. Agarwal, R.P., O'Regan, D., Saker, S.H.: Oscillation and Stability of Delay Models in Biology. Springer, New York (2014)

4. Li, T., Pintus, N., Viglialoro, G.: Properties of solutions to porous medium problems with different sources and boundary conditions. Z. Angew. Math. Phys. 70(3), 86 (2019)

5. Li, T., Viglialoro, G.: Boundedness for a nonlocal reaction chemotaxis model even in the attraction-dominated regime. Differ. Integral Equ. 34(5-6), 315-336 (2021) 
6. Viglialoro, G., Woolley, T.E.: Solvability of a Keller-Segel system with signal-dependent sensitivity and essentially sublinear production. Appl. Anal. 99(14), 2507-2525 (2020)

7. Infusino, M., Kuhlmann, S.: Infinite dimensional moment problem: open questions and applications. In: Contemp. Math., vol. 697, pp. 187-201. Am. Math. Soc., Providence (2017)

8. Ghasemi, M., Infusino, M., Kuhlmann, S., Marshall, M.: Moment problem for symmetric algebras of locally convex spaces. Integral Equ. Oper. Theory 90(3), 29 (2018)

9. Infusino, M., Kuna, T., Lebowitz, J.L., Speer, E.R.: The truncated moment problem on $\mathbb{N}_{0}$. J. Math. Anal. Appl. 452(1), 443-468 (2017)

10. Shen, J.H., Wang, Z.C.: Oscillation and asymptotic behaviour of solutions of delay differential equations with impulses. Ann. Differ. Equ. 10(1), 61-68 (1994)

11. Graef, J.R., Shen, J.H., Stavroulakis, I.P.: Oscillation of impulsive neutral delay differential equations. J. Math. Anal. Appl. $268,310-333(2002)$

12. Shen, J., Zou, Z: Oscillation criteria for first order impulsive differential equations with positive and negative coefficients. J. Comput. Appl. Math. 217, 28-37 (2008)

13. Karpuz, B., Ocalan, O.: Oscillation criteria for a class of first-order forced differential equations under impulse effects. Adv. Dyn. Syst. Appl. 7(2), 205-218 (2012)

14. Tripathy, A.K., Santra, S.S.: Characterization of a class of second-order neutral impulsive systems via pulsatile constant. Differ. Equ. Appl. 9(1), 87-98 (2017)

15. Tripathy, A.K., Santra, S.S.: Necessary and sufficient conditions for oscillation of a class of second-order impulsive systems. Differ. Equ. Dyn. Syst. (2018). https://doi.org/10.1007/s12591-018-0425-7

16. Santra, S.S., Tripathy, A.K.: On oscillatory first order nonlinear neutral differential equations with nonlinear impulses. J. Appl. Math. Comput. 59, 257-270 (2019). https://doi.org/10.1007/s12190-018-1178-8

17. Santra, S.S., Dix, J.G.: Necessary and sufficient conditions for the oscillation of solutions to a second-order neutral differential equation with impulses. Nonlinear Stud. 27(2), 375-387 (2020)

18. Tripathy, A.K., Santra, S.S.: On the forced impulsive oscillatory nonlinear neutral systems of the second-order. Nonlinear Oscil. 23(2), 274-288 (2020)

19. Tripathy, A.K., Santra, S.S.: Necessary and sufficient conditions for oscillations to a second-order neutral differential equations with impulses. Kragujev. J. Math. 47(1), 81-93 (2023)

20. Santra, S.S., Ghosh, T., Bazighifan, O.: Explicit criteria for the oscillation of second-order differential equations with several sub-linear neutral coefficients. Adv. Differ. Equ. 2020, 643 (2020)

21. Santra, S.S., Dassios, l., Ghosh, T.: On the asymptotic behavior of a class of second-order non-linear neutral differential equations with multiple delays. Axioms 9,134 (2020)

22. Santra, S.S., Majumder, D., Bhattacharjee, R., Bazighifan, O., Khedher, K., Marin, M.: New theorems for oscillations to the differential equations with mixed delays. Symmetry 13, 367 (2021)

23. Santra, S.S., Bazighifan, O., Ahmad, H., Second-Order, Y.-M.C.: Differential equation: oscillation theorems and applications. Math. Probl. Eng. 2020, 8820066 (2020)

24. Santra, S.S., Bazighifan, O., Ahmad, H., Yao, S.-W.: Second-order differential equation with multiple delays: oscillation theorems and applications. Complexity 2020, 8853745 (2020)

25. Bazighifan, O., Ruggieri, M., Scapellato, A.: An improved criterion for the oscillation of fourth-order differential equations. Mathematics 8(4), 610 (2020)

26. Bazighifan, O., Ruggieri, M., Santra, S.S., Scapellato, A.: Qualitative properties of solutions of second-order neutral differential equations. Symmetry 12(9), 1520 (2020)

27. Berezansky, L., Braverman, E.: Oscillation of a linear delay impulsive differential equations. Commun. Appl. Nonlinear Anal. 3, 61-77 (1996)

28. Diblik, J., Svoboda, Z., Smarda, Z:: Retract principle for neutral functional differential equation. Nonlinear Anal., Theory Methods Appl. 71(12), 1393-1400 (2009)

29. Santra, S.S., Alotaibi, H., Bazighifan, O.: On the qualitative behavior of the solutions to second-order neutral delay differential equations. J. Inequal. Appl. 2020, 256 (2020)

30. Diblik, J.: Positive solutions of nonlinear delayed differential equations with impulses. Appl. Math. Lett. 72, 16-22 (2017)

31. Luo, Z., Jing, Z.: Periodic boundary value problem for first-order impulsive functional differential equations. Comput. Math. Appl. 55, 2094-2107 (2008)

32. Yu, J., Yan, J.: Positive solutions and asymptotic behavior of delay differential equations with nonlinear impulses. J. Math. Anal. Appl. 207, 388-396 (1997)

33. Santra, S.S., Khedher, K.M., Moaaz, O., Muhib, A., Yao, S.-W.: Second-order impulsive delay differential systems: necessary and sufficient conditions for oscillatory or asymptotic behavior. Symmetry 13, 722 (2021). https://doi.org/10.3390/sym13040722

34. Santra, S.S., Khedher, K.M., Yao, S.-W.: New aspects for oscillation of differential systems with mixed delays and impulses. Symmetry 13, 780 (2021). https://doi.org/10.3390/sym13050780

35. Santra, S.S., Sethi, A.K., Moaaz, O., Khedher, K.M., Yao, S.-W.: New oscillation theorems for second-order differential equations with canonical and non-canonical operator via Riccati transformation. Mathematics 9, 1111 (2021). https://doi.org/10.3390/math9101111

36. Santra, S.S., Bazighifan, O., Postolache, M.: New conditions for the oscillation of second-order differential equations with sublinear neutral terms. Mathematics 9, 1159 (2021). https://doi.org/10.3390/math9111159

37. Santra, S.S. Khedher, K.M., Nonlaopon, K., Ahmad, H. New results on qualitative behavior of second order nonlinear neutral impulsive differential systems with canonical and non-canonical conditions. Symmetry 13, 934 (2021). https://doi.org/10.3390/sym13060934

38. Tripathy, A.K.: Oscillation criteria for a class of first order neutral impulsive differential-difference equations. J. Appl. Anal. Comput. 4, 89-101 (2014)

39. Berezansky, L., Domoshnitsky, A., Koplatadze, R.: Oscillation, Nonoscillation, Stability and Asymptotic Properties for Second and Higher Order Functional Differential Equations. Chapman \& Hall, Boca Raton (2020)

40. Chatzarakis, G.E., Jadlovská, I., Li, T.: Oscillations of differential equations with non-monotone deviating arguments. Adv. Differ. Equ. 2019, 233 (2019) 
41. Chatzarakis, G.E., Li, T.: Oscillation criteria for delay and advanced differential equations with nonmonotone arguments. Complexity 2018, Article ID 8237634 (2018)

42. Chatzarakis, G.E., Li, T.: Oscillations of differential equations generated by several deviating arguments. Adv. Differ. Equ. 2017, $292(2017)$

43. Agarwal, R.P., Bohner, M., Li, T., Zhang, C.: A new approach in the study of oscillatory behavior of even-order neutral delay differential equations. Appl. Math. Comput. 225, 787-794 (2013)

44. Agarwal, R.P., Zhang, C., Li, T.: Some remarks on oscillation of second order neutral differential equations. Appl. Math. Comput. 274, 178-181 (2016)

45. Bohner, M., Hassan, T.S., Li, T.: Fite-Hille-Wintner-type oscillation criteria for second-order half-linear dynamic equations with deviating arguments. Indag. Math. 29(2), 548-560 (2018)

46. Bohner, M., Li, T.: Oscillation of second-order $p$-Laplace dynamic equations with a nonpositive neutral coefficient. Appl. Math. Lett. 37, 72-76 (2014)

47. Chatzarakis, G.E., Grace, S.R., Jadlovská, I., Li, T., Tunç, E.: Oscillation criteria for third-order Emden-Fowler differential equations with unbounded neutral coefficients. Complexity 2019, Article ID 5691758 (2019)

48. Chiu, K.-S., Li, T:: Oscillatory and periodic solutions of differential equations with piecewise constant generalized mixed arguments. Math. Nachr. 292(10), 2153-2164 (2019)

49. Džurina, J., Grace, S.R., Jadlovská, I., Li, T.: Oscillation criteria for second-order Emden-Fowler delay differential equations with a sublinear neutral term. Math. Nachr. 293(5), 910-922 (2020)

50. Li, T., Rogovchenko, Y.V.: Oscillation of second-order neutral differential equations. Math. Nachr. 288(10), 1150-1162 (2015)

51. Li, T., Rogovchenko, Y.V.: Oscillation criteria for even-order neutral differential equations. Appl. Math. Lett. 61, 35-41 (2016)

52. Li, T., Rogovchenko, Y.V.: Oscillation criteria for second-order superlinear Emden-Fowler neutral differential equations. Monatshefte Math. 184(3), 489-500 (2017)

53. Li, T., Rogovchenko, Y.V.: On the asymptotic behavior of solutions to a class of third-order nonlinear neutral differential equations. Appl. Math. Lett. 105, 1-7 (2020)

54. Gyori, I., Ladas, G.: Oscillation Theory of Delay Differential Equations with Applications. Clarendon, Oxford (1991)

\section{Submit your manuscript to a SpringerOpen ${ }^{\circ}$ journal and benefit from:}

- Convenient online submission

- Rigorous peer review

Open access: articles freely available online

- High visibility within the field

- Retaining the copyright to your article

Submit your next manuscript at $\boldsymbol{~ s p r i n g e r o p e n . c o m ~}$ 